\title{
Further steps towards one culture
}

\section{Consilience: The Unity of Knowledge}

by E. O. Wilson

Knopf: 1998. Pp. 322. \$26

\section{Paul H. Harvey}

It was 1975. John Maynard Smith burst into my room. He had looked at E. O. Wilson's new book, the now infamous Sociobiology, and exclaimed that every Marxist in the Western world would be outraged by it. Maynard Smith was right, although the extent to which Wilson had anticipated the kerfuffle has never been clear.

Six major books on, Wilson brings us Consilience. Its first thesis is that the humanities, sociology, religion, ethics, art appreciation and almost everything else outside the current remit of science that has not found its analytical roots in evolutionary biology will soon do so. Its second thesis is that, once we understand how we came to be, we shall be constrained in determining where to go. Does this mean Wilson has failed to rehabilitate himself? The answer is no. The book is marked by a scientific rigour found wanting by many in his earlier works. Every step of the way he questions his assumptions, methods and tentative conclusions. Thankfully, he has not changed his vision, except by expanding its horizons.

Wilson makes a formidable argument for consilience - unifying on the basis of a common theoretical framework - of those neardisciplines that have no rational structure. The central concept underlying the book is the epigenetic rule: the idea that, because of our genetic make-up and the environment in which we develop, each individual has biases towards making certain behavioural decisions rather than others. If we can establish what those biases are and begin to understand their molecular and physiological causes, we shall be able to understand why we behave as we do. It is a massively tall order, and in terms of getting to grips with epigenetic rules, my own impression is that we have not advanced markedly in the past decade or so. Wilson still uses the examples of colour vision and incest avoidance, the empirical bulwarks of his co-authored book Genes, Mind, and Culture from 17 years ago.

But we have come a long way in understanding the functional analysis of behaviour, development and neurobiology. The book charts a marvellous path through the important empirical findings in these disciplines, placed in a framework for understanding and revealing tasks for the future that is a model of popular science writing. It also provides the strongest evidence yet for Wilson as a successful visionary. Sociobiology was an agenda as well as a review, and one

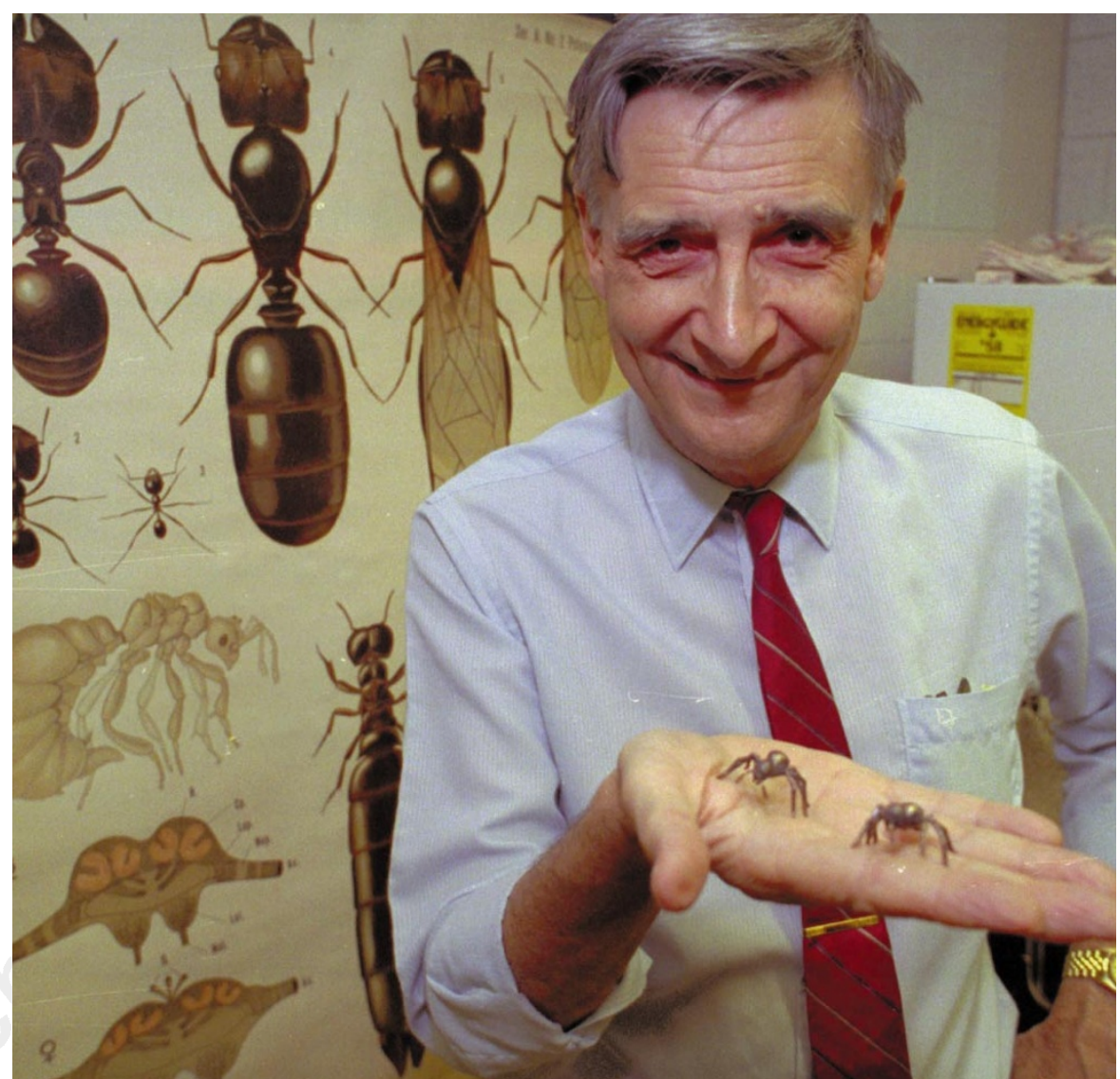

Reaching out: Wilson proposes that many disciplines will find analytical roots in evolutionary biology.

that has been largely realized. True, he got some parts wrong: his claim for the importance of trait group selection, for example. But most of it was right: evolutionary-based biological anthropology has emerged and even started to mature. He was not to know that molecular genetics would develop as it has, but its daily discoveries provide grist for his mill.

But in his new book, when discussing ethics, religion, sociology or art appreciation, he is forced to epigenetic rules viewed hazily, as through a fog (not one of his own making; that is the state of the art). The concepts are clear; if we could just summon the rules, we should reveal the proper foundations for the subjects. There is a long way to go, but we can afford to be patient because there is no competition. A proper understanding of these subjects will simply have to wait until we understand our own predispositions and the reasons for them.

In art, the few glimmers afforded by Piet Mondrian's spaced trees or the golden section point towards the rules but they cannot yet describe them, let alone trace their neural causations. (Nature's weekly art and science article is exactly what we need if we are to keep ourselves abreast of the challenge.) There are beautifully written patches of fluo- rescent prose in Wilson's treatment of these subjects. He treasures apparent insights into Palaeolithic times and minds when the cultural changes occurred that produced us. $\mathrm{He}$ describes what he imagines are moments in the lives of our ancestors, often gleaned from days in the life of contemporary bushmen.

He is now the first to admit the 'just-so' nature of his tall tales, but we all have to start somewhere. It is getting a start that matters. There is no one way to proceed in science; one just has to go at it from whatever pinhole of opportunity presents itself, thinking around and around a subject until a way in is found. This view of the scientific enterprise is well known to evolutionary biologists and ecologists because they, like astronomers, often deal with the outcome of historical processes. They try to unravel history without an evident written record; in biology the fossil record can provide occasional guidelines for some problems, just as looking light years into the past can help astronomers.

The second theme of the book may still upset the die-hards. Wilson remains a determinist in an important sense. He believes that the epigenetic rules are sufficiently hardwired that our environment of choice is itself largely predetermined. We are content to behave only in certain ways and to live in 
certain sorts of societies. A scientist wants to know why, but for the politician and the economist this limits courses of action. There will, of course, be alternatives available, but in Wilson's world they are appreciably more restricted than is generally thought. Economists do not appreciate the options because they have yet to think of them.

Wilson is a kind man, but he has no problems producing withering denouncements where they are called for. His description of our ecological plight, and his heartfelt plea for understanding and urgent action, are written with such authority, clarity and passion that I have read nothing to touch them. Nobel laureates in economics who fail to appreciate the world's finite resources and who argue for our wants in the short term rather than our needs for a sustained future are targets in this book. Evolutionary psychologists also get a timely warning. I was delighted to see one of their gurus pointing out so very dispassionately that, although Darwinian explanations abound, the theory is in its infancy and the database against which ideas are being tested remains poor.

Within this second theme, Wilson is at pains to circumscribe his own limits. Returning to art, he writes: "While biology has an important part to play in scholarly criticism, the creative arts themselves can never be locked in by this or any other discipline of science.... Works of art communicate feeling directly from mind to mind, with no intent to explain why the impact occurs. In this defining quality, the arts are the antithesis of science."

Wilson has had such a broad agenda through his career that he has frequently sought the advice of others for complementary expertise. For some of his research monographs he has collaborated with coauthors. At one end of the axis of excellence was Robert MacArthur. Fortunately, like George Oster, most of Wilson's collaborators lie close to MacArthur in this respect. And as with Sociobiology, so with Consilience - the vision is Wilson's but much of the detailed understanding of mechanism is necessarily gleaned from experts in their own fields. The responses from those working in the areas to which the clarion calls are directed may depend on how well Wilson has chosen his examples, and how accurately he has described the state of their art.

His own pool of knowledge and grasp of contemporary advances is astonishing, but even so we can expect the nitpickers to gather. I hope they will take time to evaluate the broader canvas and, where necessary, supply their own case studies in mounting whatever responses this monumental work demands. In any event, it is time to get on with the job. If we have to go through a bruising session like that following Sociobiology, I think that this time most evolutionary biologists will stand behind Wilson, albeit obliquely. He deserves no less.

Some will argue that to bring evolutionary biology and the associated epigenetic rules into such areas as art appreciation will turn us into Philistines. They should be directed to Darwin's response to the evolutionary interpretation for life itself in the last paragraph of Origin of Species. The grandeur in his view of life provided us with a new dimension for appreciating the natural world but removed none of the old. Or, as Wilson might have it, our biophilia was intensified.

Paul H. Harvey is in the Department of Zoology,

University of Oxford, South Parks Road, Oxford OX1 3PS, UK.

\section{At the limit}

\section{Impossibility}

by John D. Barrow

Oxford University Press: 1998. Pp. 279.

$£ 18.99, \$ 25$

\section{John L.Casti}

To those enamoured of the belief that the human spirit knows no bounds or limitations, a tour of twentieth-century science must be a rather depressing experience. If there is any common denominator running through the scientific breakthroughs of our age it is the idea that there are limits.

Starting with Einstein's speed limit of the Universe in a ray of light and Heisenberg's limits on what can be measured with certainty, through Gödel's limits on what can be known by following a set of rules and on to Arrow's famous result about the impossibility of a perfect democratic society, the history of modern science is riddled with logical and physical limits of all sorts.

In this illuminating, well-written account of Limits (with a capital L), John D. Barrow chronicles and explains the limits of science as a reality-generation mechanism - and why it matters.

As he carefully points out, limits to the ability of science to answer a given question about the world as we see it come in all sizes and shapes. There are political and ethical limits on how much latitude a society is willing to give to investigators to answer a question. There are practical limits on the amount of time or energy or money that can be spent in answering a question. And, finally, there are logical limits.

Although Barrow pays more than lip service to the former sorts of limitations on our ability to tease out the 'scheme of things', it's clear that his real focus is on logical limits. Rightly so, too, because ultimately these are the limits that count - at least for the scientist and scholar, if not the politician and social agitator. When one is looking for a needle in a haystack, it's of more than passing interest to know that a needle is really there to be found.

As Barrow points out, all the scientific knowledge we have about nature comes from models that we create of natural phenomena. These models, in turn, are almost always mathematical in character. It follows then that it's only a small approximation to say that the issue of logical limits to science comes down to the limits of computation, as any mathematical model can be regarded as an algorithm for processing inputs (the statement of the question and its circumstances) into an output (the answer).

This notion of scientific knowledge raises several fundamental questions. How does the mathematical model relate to the realworld phenomenon it purports to represent? Does the Turing-machine model of computation impose intrinsic limits on what we can know? What is the relationship between the computational powers of the human brain and those of our computing machines?

One question missing from the list and the book - arises from the assumption that our models of nature must necessarily be mathematical. This question is: is there an alternative to a mathematical formulation of a model of natural phenomena?

The difficulty here is that one is left perched on the horns of a dilemma. Either you use the mathematical representation of the question of concern, and then try to justify why mathematical insolubility implies the same for the model's real-world correlate. Or you forsake mathematics altogether, and then face the difficulty of trying to create a convincing real-world substitute for the mathematical notion of proof, in order to produce a knockdown argument for why the question of concern is logically unanswerable. Impossibility evades this dilemma simply by ignoring the non-mathematical alternative.

But no matter. Taken on a whirlwind tour of the mind, Gödel's theorem, quantum theory, free will, voting paradoxes, time travel, computational intractability, sandpile models, the topology of space, nanotechnology, the forces of nature, the evolution of the Universe, complexity science, computer chessplaying, percolation theory, human consciousness, economic forecasting, black holes, the Brouwer fixed-point theorem, artificial intelligence and the Heisenberg uncertainty principle, one can only wonder how Barrow can possibly make all these things fit together into a coherent story about the limits to science. Well, contrary to all expectations, he does make them fit and in only 250 pages!

So for about as good an account as you're going to get of where science stops, read this book. It won't tell you any final answer. But the journey is far more interesting - and important - than the destination.

John L. Casti is at the Santa Fe Institute, 1399 Hyde Park Road, Santa Fe, New Mexico 87501, USA. 